\title{
EXPONENTIATION IS HARD TO AVOID
}

\author{
CHRIS MILLER
}

(Communicated by Andreas R. Blass)

\begin{abstract}
Let $\mathscr{R}$ be an O-minimal expansion of the field of real numbers. If $\mathscr{R}$ is not polynomially bounded, then the exponential function is definable (without parameters) in $\mathscr{R}$. If $\mathscr{R}$ is polynomially bounded, then for every definable function $f: \mathbb{R} \rightarrow \mathbb{R}, f$ not ultimately identically 0 , there exist $c, r \in \mathbb{R}, c \neq 0$, such that $x \mapsto x^{r}:(0,+\infty) \rightarrow \mathbb{R}$ is definable in $\mathscr{R}$ and $\lim _{x \rightarrow+\infty} f(x) / x^{r}=c$.
\end{abstract}

In the following, let $\mathscr{R}:=(\mathbb{R},<, 0,1,+, \cdot, \ldots)$ be an expansion of the ordered field of real numbers. "Definable" means first-order definable in $\mathscr{R}$ with parameters from $\mathbb{R}$. A function $f: X \rightarrow \mathbb{R}, X \subseteq \mathbb{R}$, is said to be definable if its graph is definable. We may, whenever convenient, assume that we deal with totally defined functions by setting partial functions equal to 0 off their domain of definition. For the rest of this note, all functions mentioned are of one variable.

We say that $\mathscr{R}$ is polynomially bounded if, for every definable function $f$, there exists $N \in \mathbb{N}$ such that ultimately $|f(x)| \leq x^{N}$. ("Ultimately" abbreviates "for all sufficiently large positive $x$ ".) We say that $\mathscr{R}$ is $O$-minimal if the definable subsets of $\mathbb{R}$ are just the finite unions of intervals of all kinds, including singletons. (For general facts about O-minimal structures, see [PS] and [KPS].)

Given definable functions $f$ and $g$ such that ultimately $g(x) \neq 0$, we write $f \sim g$ if $\lim _{x \rightarrow+\infty} f(x) / g(x)=1$.

Theorem. Let $\mathscr{R}$ be O-minimal and not polynomially bounded. Then the exponential function is definable.

Proof. By [vdD], every definable function is ultimately differentiable and either constant or strictly monotone. Thus, the germs at $+\infty$ of definable functions form a Hardy field. In particular, every definable function has a limit in $\mathbb{R} \cup$ $\{-\infty,+\infty\}$ at $+\infty$. Since $\mathscr{R}$ is not polynomially bounded, there exists a definable function $f$ such that, for all $n \in \mathbb{N}, \lim _{x \rightarrow+\infty} f(x) / x^{n}=+\infty$. By Proposition 6 of [R], there exists a definable function $h$ with $h^{\prime} \sim f^{\prime} / f$. Since $f$ is ultimately strictly increasing, $f$ has a definable compositional inverse $f^{-1}$

Received by the editors October 19, 1992 and, in revised form, December 18, 1992; results presented at the Annual Meeting of the AMS, January 14, 1993.

1991 Mathematics Subject Classification. Primary 03C50, 03C65; Secondary 26A12. 
for large positive $x$. Then $\left(h \circ f^{-1}\right)^{\prime} \sim 1 / x$ (where $x$ denotes the identity function). Let $g=h \circ f^{-1}$, so $g$ is definable and $g^{\prime} \sim 1 / x$.

We now show that the existence of a definable function $g$ with $g^{\prime} \sim 1 / x$ implies that $\log$ is definable.

First, we claim that, for all $t>0, t \neq 1, \lim _{x \rightarrow+\infty}(g(t x)-g(x))$ is finite and nonzero.

Suppose $t>1$. By the Mean Value Theorem, for large $x$ we have

$$
(g(x t)-g(x)) /(t-1)=x g^{\prime}(\xi(x)), \quad \text { with } x<\xi(x)<x t .
$$

Note then that $1 / t<x / \xi(x)<1$. Since $\lim _{x \rightarrow+\infty} x g^{\prime}(x)=1$, ultimately we have $\left|x g^{\prime}(\xi(x))-x / \xi(x)\right|<x /(2 t \xi(x))$, and thus

$$
|(g(x t)-g(x)) /(t-1)-x / \xi(x)|<1 /(2 t) .
$$

So ultimately $(t-1) /(2 t)<g(x t)-g(x)<(t-1)(1+1 /(2 t))$. Now $x \mapsto$ $g(x t)-g(x)$ is definable, so $\lim _{x \rightarrow+\infty}(g(x t)-g(x))$ exists, and the claim holds.

For $t \in(0,1)$, note that

$$
\lim _{x \rightarrow+\infty}(g(x t)-g(x))=-\lim _{x \rightarrow+\infty}(g(x / t)-g(x)) .
$$

We have now shown that there is a definable function $G:(0,+\infty) \rightarrow \mathbb{R}$ with $G(t)=\lim _{x \rightarrow+\infty}(g(x t)-g(x))$. Note that $G(1)=0$, so $G$ is nonconstant. It is easy to check that $G(s t)=G(s)+G(t)$ for all $s, t>0$. By O-minimality, $G$ is ultimately differentiable. By elementary real analysis, we have $G(t)=G^{\prime}(1) \log t$ for all $t>0$. Since $G^{\prime}(1) \neq 0, \log$ is definable, and thus exp is as well.

Remark. The exponential function is thus even definable without parameters from $\mathbb{R}$, using the fact that the exponential function is the unique differentiable function $f: \mathbb{R} \rightarrow \mathbb{R}$ with $f(0)=1$ and $f^{\prime}=f$; see [vdD].

The first known example of an O-minimal expansion of the ordered field of real numbers that is not polynomially bounded is due to Wilkie, who has recently shown (in [W1] and [W2]) that $(\mathbb{R},<, 0,1,+, \cdot, \exp )$ is O-minimal.

Proposition. Let $\mathscr{R}$ be O-minimal and polynomially bounded. Let $f$ be definable and not ultimately identically 0 . Then there exist $c, r \in \mathbb{R}, c \neq 0$, such that $f \sim c x^{r}$ and $x \mapsto x^{r}:(0,+\infty) \rightarrow \mathbb{R}$ is definable.

Proof. Since $\mathscr{R}$ is polynomially bounded, by the remarks immediately preceding Proposition 4 of [R], there exist $c, r$ such that the desired asymptotic condition holds. Then $x \mapsto x^{r}:(0,+\infty) \rightarrow \mathbb{R}$ is definable (as noted in [P]), since, for all $x>0, \lim _{y \rightarrow+\infty} f(x y) / f(y)=x^{r}$.

\section{REFERENCES}

[vdD] L. van den Dries, Remarks on Tarski's problem concerning $(\mathbb{R},+, \cdot$, exp), Logic Colloquium 1982 (G. Lolli, G. Longo, and A. Marcja, eds.), North-Holland, Amsterdam, 1984, pp. 97-121.

[KPS] J. Knight, A. Pillay, and C. Steinhorn, Definable sets in ordered structures. II, Trans. Amer. Math. Soc. 295 (1986), 593-605.

[P] Y. Peterzil, Reducts of some structures over the reals, J. Symbolic Logic 58 (1993), 955-966.

[PS] A. Pillay and C. Steinhorn, Definable sets in ordered structures. I, Trans. Amer. Math. Soc. 295 (1986), 565-592. 
[R] M. Rosenlicht, The rank of a Hardy field, Trans. Amer. Math. Soc. 280 (1983), 659-671.

[W1] A. J. Wilkie, Model completeness results for expansions of the real field I: Restricted Pfaffian functions, preprint, 1991.

[W2] _ Model completeness results for expansions of the real field II: the exponential function, preprint, 1991.

Department of Mathematics, UNiversity OF ILlinoIs, Urbana, ILLINOIS 61801

E-mail address: miller@math.uiuc.edu 